\title{
Application of separate reinforced concrete beam model in ABAQUS
}

\author{
X.M. Chen a , J. Duan, Y.G. Li \\ China state construction technical center, Beijing, China \\ aHanee@126.com
}

\begin{abstract}
Keywords: Separate model, Reinforced concrete, Beam, ABAQUS
Abstract. In order to simplify the modeling job for analysis of reinforced concrete structures, longitudinal rebar can be simulated with equivalent box section instead of being embedded in concrete section. Separate model and monolithic model are researched by using numerical method. Numerical results show that these two models can present almost the same solutions in linear analysis, but the differences in nonlinear analysis should not be neglected. In separate model, concrete may be damaged more serious than monolithic model, but both the load bearing capacity and ductility of separate model are overestimated because the contribution of rebar has been amplified improperly. So the reinforcement ratio should be reduced properly in nonlinear analysis while using separate reinforcement model
\end{abstract}

\section{Introduction}

Most of civil engineering structures are constructed in reinforced concrete. Compared with steel structures, reinforced concrete structures are more difficult because of the mechanical property of concrete and the composite form of section. For a steel member, if slenderness ratio can be qualified, the member will present same property in both tension and compression, but concrete has quite different performance under compression and tension. Usually compression strength of concrete is times of tension strength. Concrete can keep residual strength after compression damage, but it cannot carry any tension after crack. At last, although concrete can exhibit more or less ductility, but in most situations, it must depend rebar to improve the ductility and load bearing capacity.

For frameworks of reinforced concrete, stirrups are used for improving the ductility of concrete and longitudinal rebar are embedded to carry out the tension induced by bending moment. Sometimes, shape steel or compression reinforcement may also be used to increase the load bearing capacity of section.

According to Chinese designing code, reinforcement is designed as the redundancy of load bearing capacity under frequent earthquake. Therefore, its stiffness is neglected in the section design. But the contribution of stirrup and longitudinal rebar must be taken into account in the analysis under expected rare earthquake, where the constraint effect of stirrup can be formulated into the constitutive relation of concrete[1] and the stiffness of longitudinal rebar must be presented by element model.

Longitudinal rebar can be embedded into the section as monolithic model or simulated as a separate element. Although by embedding rebar into the section, FEA analysis can present more accurate results, but in order to simplify the modeling job, engineers prefer to use separate reinforcement model $[2,3,4]$. Most of these researches are focused on 3D model, which cannot be extended to the analysis of high-rise buildings.

In building structures, shell elements are used to simulate shear walls and slabs, beam elements are used to simulate frameworks. Rebar can be embedded into shear walls or slabs for both implicit method and explicit method in ABAQUS, and the stiffness of rebar layers are formulated with isoparametric mapping method[5]. But for beam elements, embedding of rebar is not available in explicit method, which is more powerful than implicit method for analysis of nonlinear time-history. Then engineers have to develop new beam element to overcome this problem[6]. On the other hand, concrete and rebar can be simulated with separate beam elements respectively. In separate model, the bond-slip behavior is neglected, and except for the concrete element, new steel elements with box 
section are introduced instead of rebar. This box section has the same area as the longitudinal reinforcement area.

A simple framework under horizontal displacement load is analyzed in this paper. The results of section bending moment, nodal drilling angle, compression damage of concrete and plastic strain of steel are used for comparison between monolithic model and separate model. This can help engineers to modeling for reinforced concrete structures in analysis of nonlinear time-history under expected rare earthquake.

\section{Monolithic model}

By using monolithic model, rebar can be embedded into the concrete section in ABAQUS as shown in Fig.1.

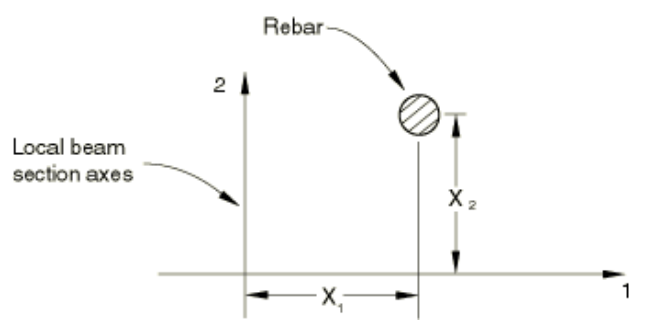

Fig.1 Rebar in beam element

And the definition is as follows:

*REBAR, ELEMENT=BEAM, MATERIAL=mat, NAME=name

Each date line can define a single rebar. By the material name, ABAQUS can index its constitutive relationship in section numerical integration. Except for the integration points appointed for concrete section, additional integration points will be appointed for each rebar as shown in Fig.2.

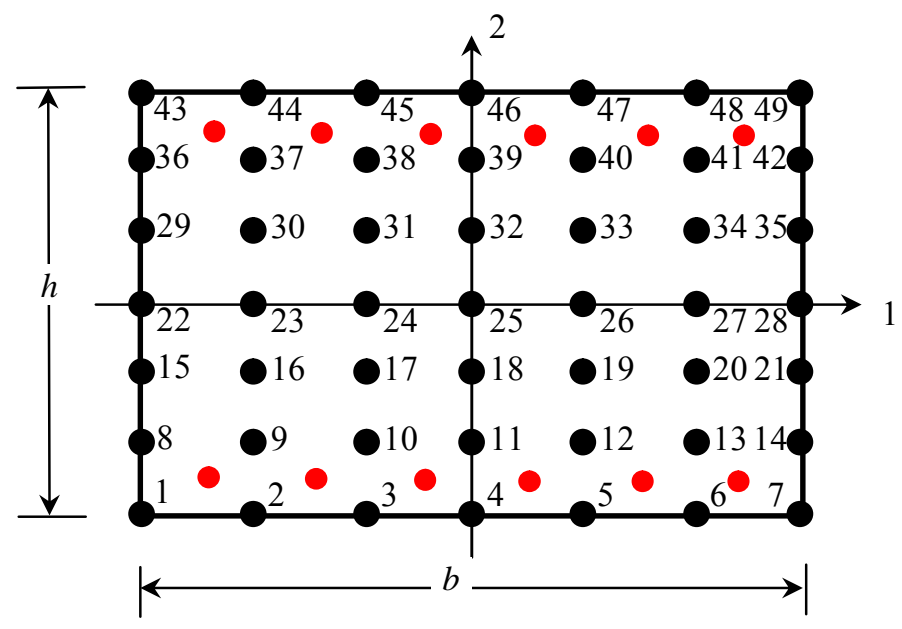

Fig.2 Integration points of rectangular section of reinforced concrete

As a fiber, a single rebar can carry both tension and compression because of the constraint effect of concrete. By integration, all the rebar can carry bending moment like concrete section as follows:

$$
\begin{aligned}
& M_{x}=\sum_{i=1}^{n} A_{i} h_{y} \sigma_{i} \\
& M_{y}=\sum_{i=1}^{n} A_{i} h_{x} \sigma_{i} \\
& N=\sum_{i=1}^{n} A_{i} \sigma_{i}
\end{aligned}
$$

where 
$A_{i}$ is the area of each rebar;

$h_{x}$, hy are the distance to neutral axis of each rebar;

$\sigma_{i}$ is the axial stress of each rebar.

\section{Separate model}

In separate beam model, longitudinal rebar are transformed to a box section with equivalent section area(Fig.4). Concrete is reinforced at top and bottom of the section. The area of top flange and bottom is equal to the area of rebar respectively:

$$
\begin{aligned}
& A_{u}=\sum_{i=1}^{n_{u}} A_{u i} \\
& A_{d}=\sum_{i=1}^{n_{d}} A_{d i}
\end{aligned}
$$

Where

$A_{u i}$ is the area of a single rebar at the top,

$A_{d i}$ is the area of a single rebar at the bottom,

$n_{u}$ is the number of rebar at the top,

$n_{d}$ is the number of rebar at the bottom,

The thickness of web is assumed to be a small value as $1 \mathrm{~mm}$. Because of the constraint effect of concrete, rebar can keep free from buckling, thus warping of this thin-wall section can be neglected. On the other hand, the element of rebar should be simulated with beam element but not truss element for the reason of bending stiffness.
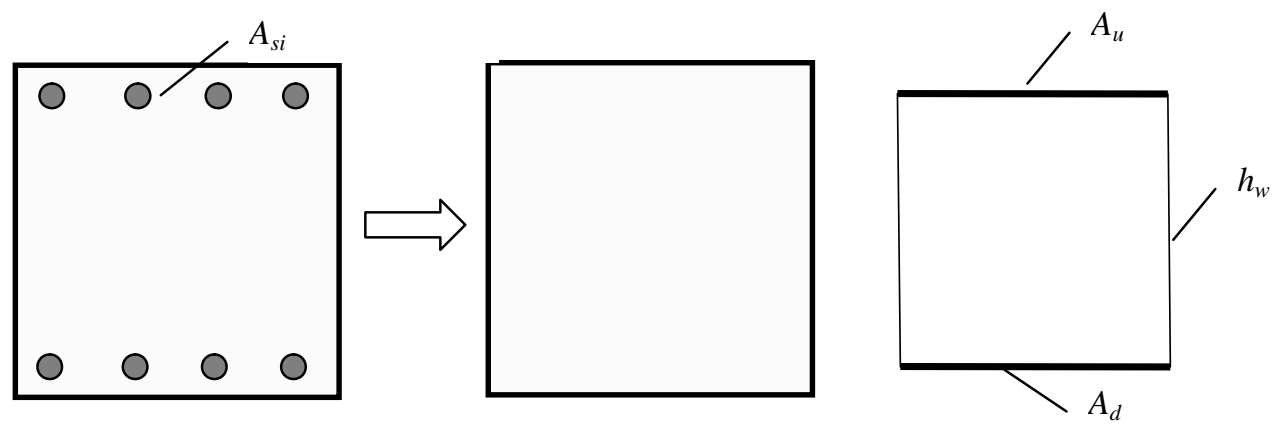

Fig.3 Equivalence of reinforced concrete section

The default integration points for the equivalent steel box element are shown in Fig.4:

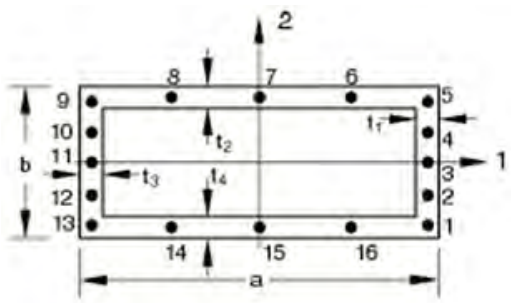

Fig.4 Default integration points of box section

\section{Numerical example}

A framework shown in Fig. 5 is analyzed. height is $3.3 \mathrm{~m}$, span is $6.0 \mathrm{~m}$, elemental length is $0.5 \mathrm{~m}$, and cubic element B33 is used. The area of each rebar is $3.8 \mathrm{~cm} 2$ and the integration points for concrete section is $7 \times 7$. Separate model is used for columns, monolithic and separate models are used 
for beam for comparison. Density of material is assumed to be zero. Displacement load is applied at the top of columns from 0 to $5 \mathrm{~cm}$ in one second. The load bearing capacity and compression damage at leftmost section of beam are researched for comparing the difference of these two models. The results of section integration points on local axis y(from bottom to top:4,11,18,25,32,39,46) are listed in Fig.7 and Fig.8.
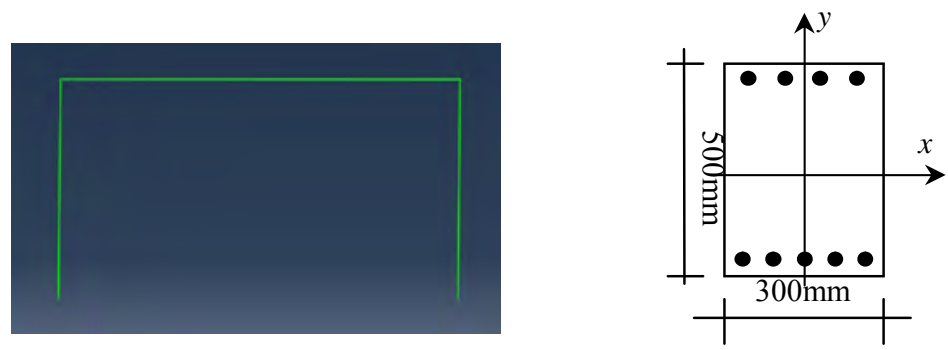

Fig.5 Structural model

Results of bending moment and drilling angle of beam left end for both linear analysis and nonlinear analysis are shown in Fig.6. The results of concrete section and equivalent steel box section are also presented for separate model. As shown in Fig.6(a), solutions of separate model is almost the same as monolithic model. It is quite different in nonlinear analysis as shown in Fig.6(b). The difference of curves can be neglected before the peak value, but it is obvious that separate model overestimated the maximum load bearing capacity and ductility.

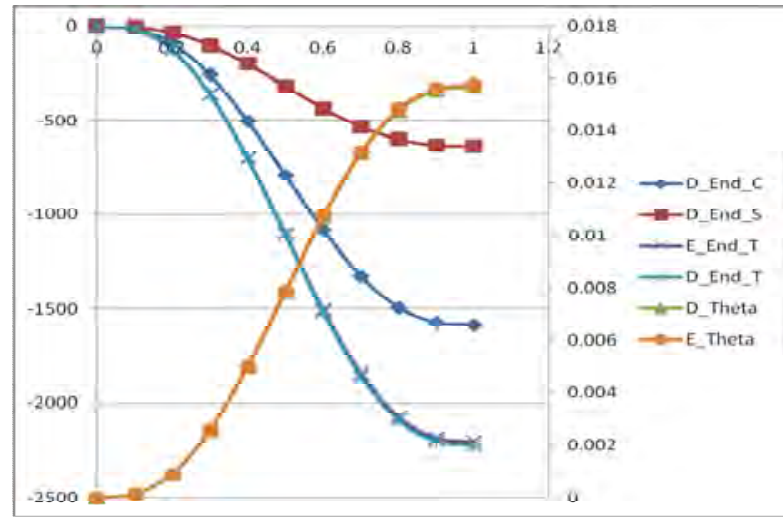

(a) Linear analysis

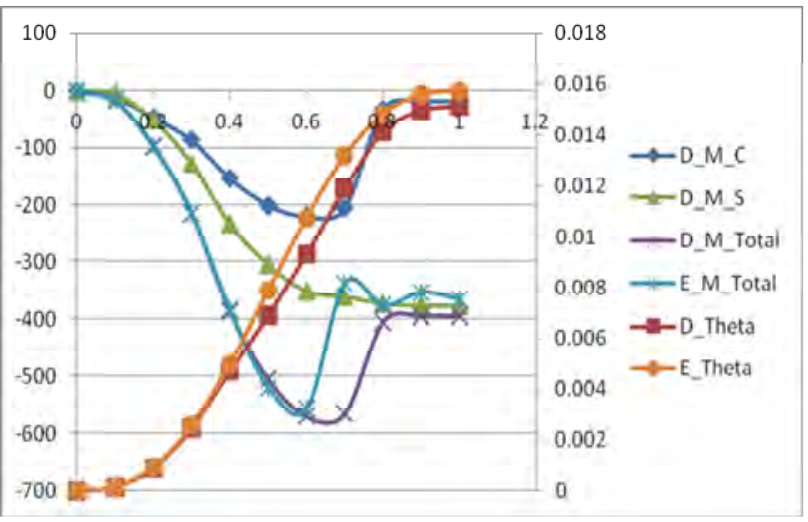

(b) Nonlinear analysis

Fig.6 Comparison of Moment and drilling angle of monolithic model and separate model

Results of compression damage at left end of beam are shown in Fig.7. These two models presented almost the same maximum values of compression damage, but the distribution is quite different. For integration point 32 , the compression damage is up to 0.7 , but it is only about 0.1 in monolitic model. It can be seen that the height of compression zone in separate model is overestimated.

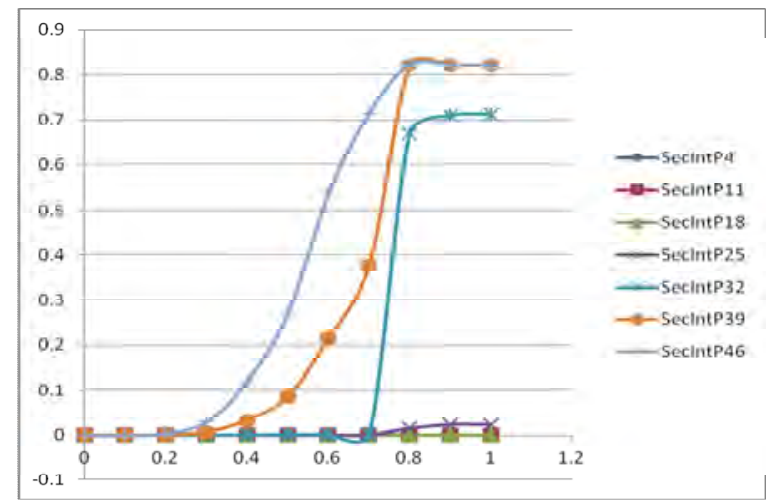

(a) Separate model

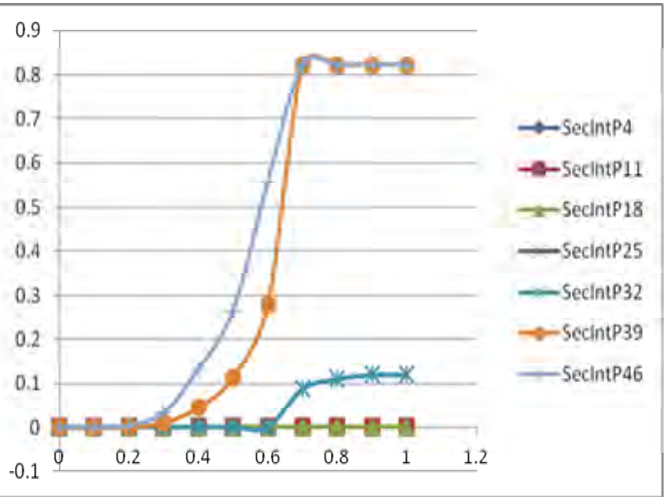

(b) Monolithic model

Fig.7 Compression damage of concrete

Results of concrete compression strength are shown in Fig.8. There are two integration points which strength dereased because of damage for both monolithic model and separate model. The 
integration points in compression show that the offset of compression area in monolithic model is much more significant than separate model.

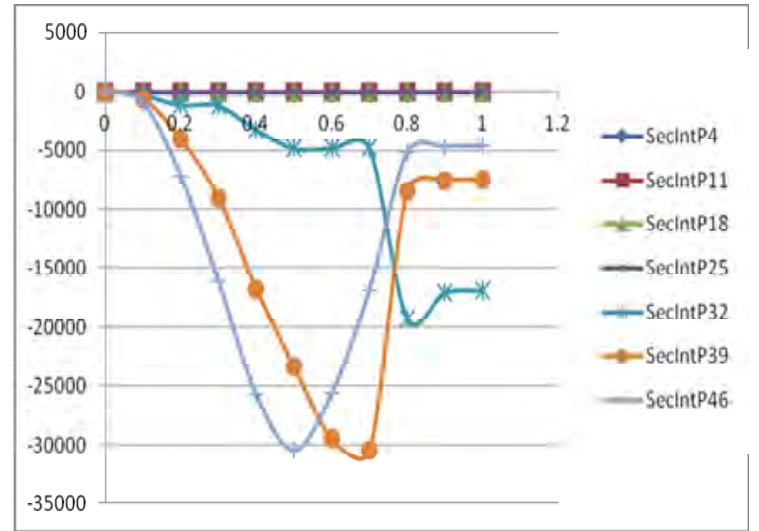

(a) Separate model

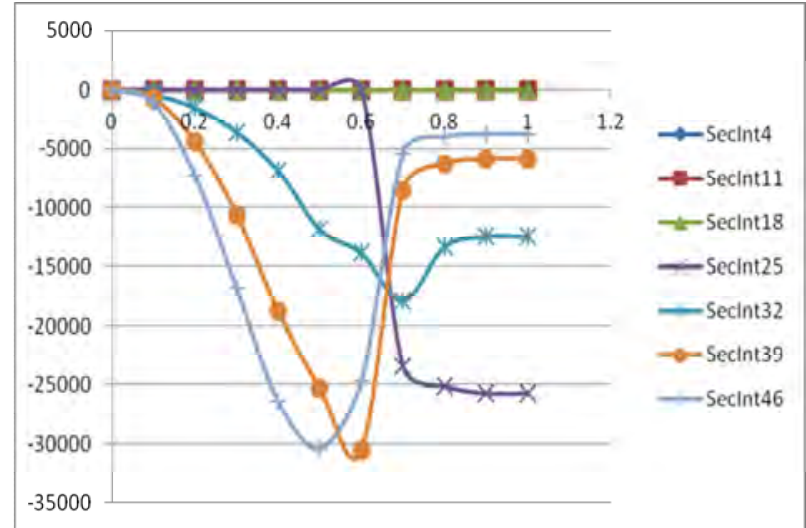

(b) Monolithic model

Fig.8 Compression strength of concrete

\section{Conclusions}

Separate model and monolithic model are researched for reinforced concrete framework by using numerical method. Numerical results show that these two models can present almost the same solutions in linear analysis, but there are some differences in nonlinear analysis, for example, concrete may be damaged more seriously in separate model than monolithic model, on the other hand, the load bearing capacity and ductility of separate model are also overestimated, it means the contribution of rebar has been amplified improperly. So it is recommended that the reinforcement ratio should be reduced in nonlinear analysis while using separate reinforcement model.

\section{References}

[1] Code for design of concrete structures. China architecture \& building press, Beijing, China (2010). (In Chinese)

[2] Hou Jianhua, Lu Bing, Wang Zhi. Research on separate model for reinforcement concrete framework. Henan building materials. 2014(2):18

[3] Zhang Jie, Gao Rongyu. Finite element analysis between the separate and monolithic reinforcement concrete model. Journal of Anhui Institute of Architecture \& Industry.. 2013,21(4):5

[4] Mao Ying, Fan Jiang, Tao Yan. Finite element analysis of reinforced concrete structures using discrete model. Engineering Mechanics(Supplement). 2003: 595

[5] ABAQUS Inc(2006). ABAQUS UserManual, V6. 5. 5.

[6] Feng Lijuan, Shang Xiaojiang, Xu Ziguo. Static and dynamic elasto-plastic analysis of structures based on ABAQUS. Earthquake Resistant Engineering and Retrofitting. 2008,30(5): 14 